\title{
A transversely isotropic medium with a tilted symmetry axis normal to the reflector ${ }^{a}$
}

${ }^{a}$ Published in Geophysics (Letters), 75, A19-A24 (2010)

Tariq Alkhalifah, King Abdulah University of Science and Technology, and Paul Sava, Center for Wave Phenomena, Colorado School of Mines ${ }^{1}$

\begin{abstract}
The computational tools for imaging in transversely isotropic media with tilted axes of symmetry (TTI) are complex and in most cases do not have an explicit closed-form representation. As discussed in this paper, developing such tools for a TTI medium with tilt constrained to be normal to the reflector dip (DTI) reduces their complexity and allows for closed-form representations. We show that, for the homogeneous case zero-offset migration in such a medium can be performed using an isotropic operator scaled by the velocity of the medium in the tilt direction. We also show that, for the nonzero-offset case, the reflection angle is always equal to the incidence angle, and thus, the velocities for the source and receiver waves at the reflection point are equal and explicitly dependent on the reflection angle. This fact allows us to develop explicit representations for angle decomposition as well as moveout formulas for analysis of extended images obtained by wave-equation migration. Although setting the tilt normal to the reflector dip may not be valid everywhere (i.e., salt flanks), it can be used in the process of velocity model building where such constrains are useful and typically used.
\end{abstract}

\section{INTRODUCTION}

In recent years and with the increasing emphasis on high resolution and the availability of better computing devices, anisotropic-media treatment of seismic data is becoming part of normal operations rather than the exception. This preference is fueled by the recent observed improvements in, for example, Gulf of Mexico images when anisotropy is included in the process (Zhou et al., 2004; Huang et al., 2008). A special anisotropy, a transversely isotropic medium with a tilt in the axis of symmetry, is especially convenient in approximating the features of the medium in such regions, and provides good imaging results.

Anisotropy characterization comes in many flavors approximating all sorts of phenomena present in the subsurface by introducing directional preferences in the velocity field that accommodates such phenomena. Whether we are dealing with the natural processes of sedimentation and gravity, especially in shales, or the ever localized vertical fractures

\footnotetext{
11e-mail: tkhalfah@kacst.edu.sa,psava@mines.edu
} 
(some also non-vertical), we can find an anisotropy that will approximate the processes influence and produce wavefields that can accurately represent wave propagation in such media. The inclusion of anisotropy into the imaging and velocity model-building process evolved through the years. As expected, we started by looking into using the simplest of the anisotropies, that is elliptical anisotropy, to handle depth shortcomings of the isotropic assumption (Ball, 1995; Ohlsen and MacBeth, 1999), despite its impracticality. However, the size of the consistent nonhyperbolicity forced some to use a slightly more complex model and yet more practical (Alkhalifah and Larner, 1994), that is the transversely isotropic (TI) media with a vertical symmetry axis (VTI). Though this model proved resilient in many areas of the subsurface (Alkhalifah, 1997; Martinez and Lee, 2002), the dips of layers near salt flanks seemingly required additional degrees of freedom provided by the tilt of the symmetry axis in the transversely isotropic medium (Isaac and Lawton, 2004).

The vertical and normal moveout (NMO) velocities, as well as the nonhyperbolic dimensionless parameter, $\eta$, define the anisotropy aspects of the TI model for P-waves, at least to the accuracy required for prestack imaging (Alkhalifah and Tsvankin, 1995). If the symmetry axis is vertical no other parameters are needed to define the TI model. However, since the stratification in the Earth subsurface is not always horizontal, we can expect the symmetry axis to have some deviation from the vertical especially around salt-body flanks. For TI media with a tilt in the axis of symmetry, two additional parameters that describe the tilt in 3D, are needed to fully characterize acoustic wave propagation. These two parameters are often estimated by assuming that the tilt direction is normal to the medium structure or in the direction of the velocity gradient (Alkhalifah and Bednar, 2000; Audebert et al., 2006). Setting the tilt normal to the dip direction has been convenient and practical. Audebert et al. (2006) realized through numerical experimentation that constraining the tilt of the symmetry axis to the structure, in what they referred to as structurally conformable TI (STI) media, results in simplifications in the parameter dependency in which the short-spread focusing becomes decoupled from long-spread behavior. In fact, setting the tilt normal to the dip results in simplified equations for data analysis, as we discussed here.

In this paper, we show explicitly that when we constrain the tilt to be normal to the reflector dip, the behavior of plane waves around the scattering point is explicitly represented by closed-form relations. In fact, the reflection angles for the source and the receiver rays are always equal. Thus, the key is to include this constraint as part of the process, whether it is migration or angle-gather development. As a result, we call the medium dip-constrained TI (DTI) to stress the concept of using this constraint as part of the process as opposed to relating it to the structure of the model. We show that key equations are simplified by this constraint, which will help in boosting the efficiency of such processes. In fact, the DTI model makes processes such as angle-gather decomposition that depend on development around the scattering point simpler than the VTI model.

\section{DIP-CONSTRAINED TTI MEDIA}

To appreciate the simplification attained from this constraint, we initially restrict our discussions to a homogeneous medium. In this case, the zero-offset isochron, which is repre- 


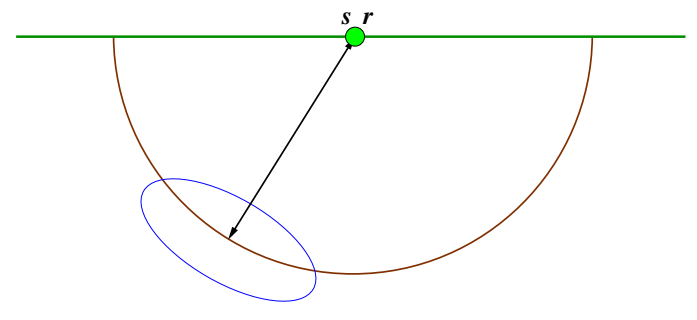

(a)

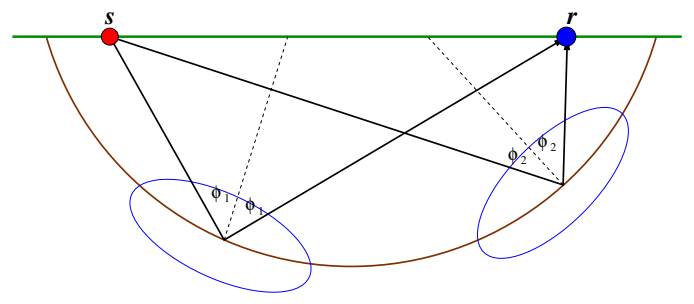

(b)

Figure 1: (a) A schematic representation of the tilted transverse isotropy isochron for zerooffset in which the tilt is constrained to the normal of the isochron - the isochron is circular. (b) A similar representation for the non-zero offset case. The angle $\phi$ is the group angle which is equal for the incident and reflected rays, but differs along the isochron.

sentative of the equal traveltime surface, is spherical in shape, equivalent to the isotropic medium isochron, with a radius governed by the velocity in the tilt direction, $v_{T}$, as follows:

$$
r(\mathbf{x})=v_{T} t(\mathbf{x})
$$

where $t$ is the time along the wavefront and $\mathbf{x}=\{x, y, z\}$ represents space coordinates. This convenient assertion is only true if we constrain the tilt axis to the direction normal to the reflector dip, and thus the group velocity equals the phase velocity equals the velocity along the tilt. Figure 1(a) shows a schematic plot of the zero-offset isochron with two representative examples of tilt direction that are constrained to be orthogonal to the isochron surface. Though such a medium do not physically exist, it is assumed here in the context of a process, and thus what matters is the local action of the isochron on the reflection, which is similar to the isotropic case.

For non zero-offset case, the traveltime isochron is constrained by the double-squareroot (DSR) formula (Claerbout, 1995). Thus, the total traveltime, $t$, is a combination of traveltimes from the source $\mathbf{s}$ located at $\left(s_{x}, s_{y}\right)$, and the receiver $\mathbf{r}$ located at $\left(r_{x}, r_{y}\right)$ to an image point in the subsurface at location $\mathrm{x}$ and is given by the expression

$$
\begin{aligned}
t & =\sqrt{\frac{\left(s_{x}-x\right)^{2}+\left(s_{y}-y\right)^{2}+z^{2}}{v_{g}^{2}(\phi)}} \\
& +\sqrt{\frac{\left(r_{x}-x\right)^{2}+\left(r_{y}-y\right)^{2}+z^{2}}{v_{g}^{2}(\phi)}}
\end{aligned}
$$

where $v_{g}(\phi)$ is the group velocity as a function of group angle $\phi$. From Figure 1(b), and considering, for simplicity, that the incident and reflected rays are confined to the vertical 
plane, $\phi$ can be evaluated geometrically as follows:

$$
\begin{aligned}
\phi & =\frac{1}{2} \cos ^{-1} \frac{z}{\sqrt{\left(s_{x}-x\right)^{2}+\left(s_{y}-y\right)^{2}+z^{2}}} \\
& +\frac{1}{2} \cos ^{-1} \frac{z}{\sqrt{\left(r_{x}-x\right)^{2}+\left(r_{y}-y\right)^{2}+z^{2}}},
\end{aligned}
$$

otherwise we have to project the angles to the plane that constrains the incident and reflected rays. However, evaluating $v_{g}(\phi)$ in complex media is complicated with no closedform representation. An alternative is to rely on the phase angle by using plane waves and the Fourier decomposition.

If we reformulate the DSR equation in terms of changes in time, and thus, focus on the plane-wave relation we end up with the following DSR formula:

$$
\frac{\partial t}{\partial z}=\sqrt{\frac{1}{v^{2}(\theta)}-\left(\frac{\partial t}{\partial r}\right)^{2}}+\sqrt{\frac{1}{v^{2}(\theta)}-\left(\frac{\partial t}{\partial s}\right)^{2}}
$$

where now $v$ is the phase velocity and has a closed form representation in terms of the phase angle $\theta$ given by the acoustic approximation (Alkhalifah, 1998) as follows:

$$
\begin{aligned}
v^{2}(\theta) & =\frac{1}{2}\left(v^{2}(2 \eta+1) \sin ^{2} \theta+v_{T}^{2} \cos ^{2} \theta\right) \\
& +\frac{1}{4} \sqrt{a \sin ^{4} \theta+b \sin ^{2}(2 \theta)+c \cos ^{4} \theta}
\end{aligned}
$$

where $a=4 v^{4}(2 \eta+1)^{2}, b=2 v^{2} v_{T}^{2}(1-2 \eta), c=4 v_{T}^{4}, v$ is the NMO velocity with respect to the tilted symmetry axis, and $\eta$ is the anisotropy parameter relating the NMO velocity to the velocity normal to the tilt. The angle $\theta$ in equation 4 is measured from the tilt direction and will also be given by the angle gather as part of the process of downward continuation as we will see later.

Thus, in the non-zero offset case the isochron depends on angle, but it is a single angle for both source and receiver rays and we do not have to worry about relating the two angles, as is the case in VTI and general TTI media. This provides us with analytical relations for plane waves at the reflection point. In this case, both the source and receiver waves have the same wave group velocity that differs along the non-zero offset isochron. In fact, for the zero-dip part of the isochron the reflection angle is at its maximum reducing to zero for a vertical reflector, as seen in Figure 1(b).

Next, we formulate the extended imaging condition, necessary for angle-gather development, for the DTI model. As shown in this section, angle gathers are also necessary for an explicit formulation of downward continuation in a DTI model. 


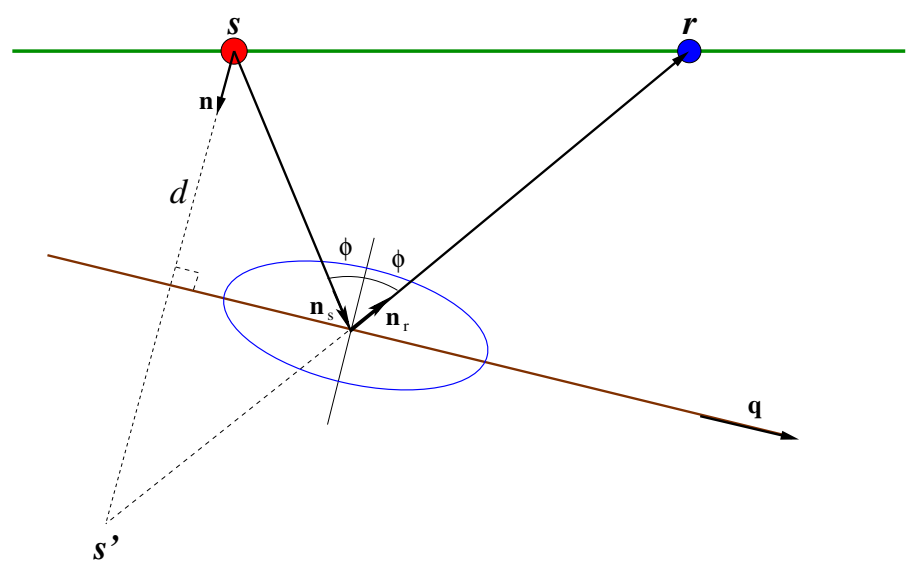

Figure 2: A schematic plot of the reflection geometry for a tilted transversely isotropic medium with a tilt in the dip direction. The incident and reflection angles are the same given by the group angle $\phi$. Here, $s$ and $r$ correspond, respectively, to the source and receiver locations, $d$ is the distance between the source and the reflector in the direction given by unit vector $\mathbf{n}$ normal to the reflector with direction described by unit vector $\mathbf{q}$, and $\mathbf{n}_{\mathrm{s}}$ and $\mathbf{n}_{\mathrm{r}}$ are, respectively, the unit vector directions for each of the source and receiver rays with ray angle $\phi$ measured from the normal to the reflector.

\section{EXTENDED IMAGING CONDITION}

Conventional seismic imaging methods share the assumption of single scattering at discontinuities in the subsurface. Under this assumption, waves propagate from seismic sources, interact with discontinuities and return to the surface as reflected seismic waves. We commonly speak about a "source" wavefield, originating at the seismic source and propagating in the medium prior to any interaction with discontinuities, and a "receiver" wavefield, originating at discontinuities and propagating in the medium to the receivers (Berkhout, 1982; Claerbout, 1985). The two wavefields kinematically coincide at discontinuities. Any mismatch between the wavefields indicates inaccurate wavefield reconstruction typically assumed to be due to inaccurate velocity. In this context, we do not need to make geometrical assumptions about up- or down-going propagation, since waves can move in any direction as long as they scatter only once. We also do not need to make any assumption about how we reconstruct those two wavefields as long as the wave-equation used accurately describes wave propagation in the medium under consideration.

We can formulate imaging as a process involving two steps: the wavefield reconstruction and the imaging condition. The key elements in this imaging procedure are the source and receiver wavefields, $u_{s}$ and $u_{r}$, which depend on space $\mathrm{x}$ and time $t$. A conventional crosscorrelation imaging condition (cIC) based on the reconstructed wavefields can be formulated as the zero lag of the crosscorrelation between the source and receiver wavefields (Claerbout, 1985):

$$
r(\mathbf{x})=\sum_{\text {shots }} \sum_{\omega} \overline{u_{s}(\mathbf{x}, \omega)} u_{r}(\mathbf{x}, \omega),
$$


where $r$ represents the migrated image and the over-line represents complex conjugation. This operation exploits the fact that portions of the wavefields match kinematically at subsurface positions where discontinuities occur.

An extended imaging condition preserves in the output image certain acquisition (e.g., source or receiver coordinates) or illumination (e.g., reflection angle) parameters (Clayton and Stolt, 1981; Claerbout, 1985; Stolt and Weglein, 1985; Weglein and Stolt, 1999). In shot-record migration, the source and receiver wavefields are reconstructed on the same computational grid at all locations in space and all times or frequencies, therefore there is no a-priori separation that can be transferred to the output image. In this situation, the separation can be constructed by local translations between the two wavefields, either in space (Rickett and Sava, 2002; Sava and Fomel, 2005), or in time (Sava and Fomel, 2006) or in space and time. This separation essentially represents local crosscorrelation lags between the source and receiver wavefields. Thus, an extended crosscorrelation imaging condition (eIC) defines the image as a function of space and crosscorrelation lags in space $\lambda$ and time $\tau$ :

$$
r(\mathbf{x}, \boldsymbol{\lambda}, \tau)=\sum_{\text {shots }} \sum_{\omega} e^{2 i \omega \tau} \overline{u_{s}(\mathbf{x}-\boldsymbol{\lambda}, \omega)} u_{r}(\mathbf{x}+\boldsymbol{\lambda}, \omega)
$$

Equation 6 represents a special case of equation 7 for $\lambda=0$ and $\tau=0$. The eIC defined by equation 7 can be used to analyze the accuracy of wavefield reconstruction.

\section{MOVEOUT ANALYSIS}

If we restrict the observation to the immediate vicinity of the reflection point, which means that we consider the moveout surface in a small range of lags, we can approximate the typical irregular wavefront in complex media by a plane, although the shapes of wavefronts are arbitrary in heterogeneous media. Following the derivation of Yang and Sava (2009) and using the geometry shown in Figure 2, the source and receiver plane waves are described by:

$$
\begin{aligned}
\mathbf{n}_{\mathbf{s}} \cdot \mathbf{x} & =v(\theta) t \\
\mathbf{n}_{\mathbf{r}} \cdot(\mathbf{x}-2 d \mathbf{n}) & =v(\theta) t
\end{aligned}
$$

where $\mathbf{n}_{\mathbf{s}}$ and $\mathbf{n}_{\mathbf{r}}$ are the unit direction vectors of the source and receiver plane waves, respectively, $\mathbf{n}$ is the unit vector orthogonal to the reflector at the image point, and vector $\mathbf{x}$ indicates the image point position. $v$ is defined as the phase velocity in the locally homogeneous medium around the reflection point, and thus it is identical for both wavefields. $\theta$ is half the scattering angle (reflection angle).

We can also obtain the shifted source and receiver plane waves by introducing the space- 
and time-lags

$$
\begin{aligned}
\mathbf{n}_{\mathbf{s}} \cdot(\mathbf{x}+\boldsymbol{\lambda}) & =v(\theta)(t+\tau), \\
\mathbf{n}_{\mathbf{r}} \cdot(\mathbf{x}-2 d \mathbf{n}-\boldsymbol{\lambda}) & =v(\theta)(t-\tau) .
\end{aligned}
$$

Solving the system of equations 10-11 leads to the expression

$$
\left(\mathbf{n}_{\mathbf{s}}-\mathbf{n}_{\mathbf{r}}\right) \cdot \mathbf{x}=2 v(\theta) \tau-\left(\mathbf{n}_{\mathbf{s}}+\mathbf{n}_{\mathbf{r}}\right) \cdot \boldsymbol{\lambda}-2 d \mathbf{n}_{\mathbf{r}} \cdot \mathbf{n},
$$

which characterizes the moveout function (surface) of space- and time-lags at a commonimage point.

Furthermore, we have the following relations for the reflection geometry:

$$
\begin{aligned}
& \mathbf{n}_{\mathbf{s}}-\mathbf{n}_{\mathbf{r}}=2 \mathbf{n} \cos \theta \\
& \mathbf{n}_{\mathbf{s}}+\mathbf{n}_{\mathbf{r}}=2 \mathbf{q} \sin \theta
\end{aligned}
$$

where $\mathbf{n}$ and $\mathbf{q}$ are unit vectors normal and parallel to the reflection plane, respectively, and $\theta$ is the reflection angle. Vector $\mathbf{q}$ characterizes the line representing the intersection of the reflection and the reflector planes. Combining equations 12-14, we obtain the moveout function for plane waves:

$$
z(\boldsymbol{\lambda}, \tau)=d_{0}-\frac{\tan \theta(\mathbf{q} \cdot \boldsymbol{\lambda})}{n_{z}}+\frac{v(\theta) \tau}{n_{z} \cos \theta} .
$$

The quantity $d_{0}$ is defined as

$$
d_{0}=\frac{d-(\mathbf{c} \cdot \mathbf{n})}{n_{z}},
$$

and represents the depth of the reflection corresponding to the chosen common-image gather (CIG) location. This quantity is invariant for different plane waves, thus assumed constant here. The vector $\mathbf{c}$ is along the Earth's surface given by $(x, y, 0)$.

When incorrect velocity is used for imaging, and thus, an inaccurate reflection angle is assumed, based on the analysis in the preceding section, we can obtain the moveout function

$$
z(\boldsymbol{\lambda}, \tau)=d_{0 f}-\frac{\tan \theta_{m}\left(\mathbf{q}_{m} \cdot \boldsymbol{\lambda}\right)}{n_{m z}}+\frac{v_{m}\left(\theta_{m}\right)\left(\tau-t_{d}\right)}{n_{m z} \cos \theta_{m}},
$$

where $d_{0 f}$ is the focusing depth of the corresponding reflection point, $v_{m}$ is the migration velocity, $t_{d}$ is the focusing error, $\mathbf{n}_{m}$ and $\mathbf{q}_{m}$ are vectors normal and parallel to the migrated reflector, respectively. Equation 17 describes the extended images moveout for a single seismic experiment and it is essentially identical to the similar formula obtained by Yang and Sava (2009) for isotropic media, but for using the phase velocity instead of the isotropic velocity. 


\section{ANGLE DECOMPOSITION}

In downward continuation methods, theoretical analysis of angle gathers can be reduced to analyzing the geometry of reflection in the simple case of a dipping reflector in a locally homogeneous medium (Sava and Fomel, 2005). The behavior of plane waves in the vicinity of the reflection point is sufficient for deriving relationships for local reflection traveltime derivatives (Goldin, 1986). The geometry of the reflection ray paths is depicted in Figure 2.

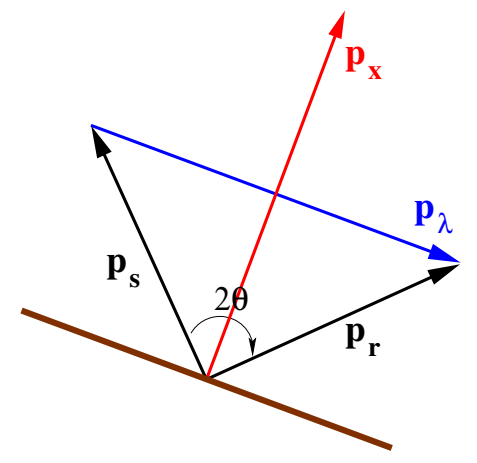

Figure 3: A schematic plot depicting the relation between source and receiver rayparameter vectors $\left(\mathbf{p}_{\mathbf{s}}\right.$ and $\left.\mathbf{p}_{\mathbf{r}}\right)$ and that of the space-lag and position $\left(\mathbf{p}_{\boldsymbol{\lambda}}\right.$ and $\left.\mathbf{p}_{\mathbf{x}}\right)$. The angle $\theta$ corresponds to the phase angle direction of the plane wave.

Using the standard notations for the source and receiver coordinates: $\mathbf{s}=\mathbf{x}+\boldsymbol{\lambda}$ and $\mathbf{r}=\mathbf{x}-\boldsymbol{\lambda}$, the traveltime from a source to a receiver is a function of all spatial coordinates of the seismic experiment $t=t(\mathbf{x}, \boldsymbol{\lambda})$. Differentiating $t$ with respect to all components of the vectors $\mathbf{x}$ and $\boldsymbol{\lambda}$, and using the standard notations to represent slownesses $\mathbf{p}_{\alpha}=\nabla_{\alpha} t$, where $\alpha=(\mathbf{x}, \boldsymbol{\lambda}, \mathbf{s}, \mathbf{r})$, we can write:

$$
\begin{aligned}
& \mathbf{p}_{\mathbf{x}}=\mathbf{p}_{\mathbf{r}}+\mathbf{p}_{\mathbf{s}} \\
& \mathbf{p}_{\boldsymbol{\lambda}}=\mathbf{p}_{\mathbf{r}}-\mathbf{p}_{\mathbf{s}}
\end{aligned}
$$

By analyzing the geometric relations of various vectors at an image point (Figures 3), we can write the following trigonometric expressions:

$$
\begin{aligned}
& \left|\mathbf{p}_{\boldsymbol{\lambda}}\right|^{2}=\left|\mathbf{p}_{\mathbf{s}}\right|^{2}+\left|\mathbf{p}_{\mathbf{r}}\right|^{2}-2\left|\mathbf{p}_{\mathbf{s}}\right|\left|\mathbf{p}_{\mathbf{r}}\right| \cos (2 \theta), \\
& \left|\mathbf{p}_{\mathbf{x}}\right|^{2}=\left|\mathbf{p}_{\mathbf{s}}\right|^{2}+\left|\mathbf{p}_{\mathbf{r}}\right|^{2}+2\left|\mathbf{p}_{\mathbf{s}}\right|\left|\mathbf{p}_{\mathbf{r}}\right| \cos (2 \theta) .
\end{aligned}
$$

Defining $\mathbf{k}_{\mathbf{x}}$ and $\mathbf{k}_{\boldsymbol{\lambda}}$ as the position and lag (or offset) wavenumber vectors, we can replace $\mathbf{p}=\mathbf{k} / \omega$. Using the trigonometric identities

$$
\begin{aligned}
& 1-\cos (2 \theta)=2 \sin ^{2}(\theta), \\
& 1+\cos (2 \theta)=2 \cos ^{2}(\theta),
\end{aligned}
$$

and assuming $\left|\mathbf{p}_{\mathbf{s}}\right|=\left|\mathbf{p}_{\mathbf{r}}\right|=s(\theta)$, where $s(\theta)=1 / v_{p}(\theta)$ is the phase slowness as a function 
of phase angle at an image location, we obtain the following relations:

$$
\begin{array}{r}
\left|\mathbf{k}_{\boldsymbol{\lambda}}\right|^{2}=(2 \omega s(\theta) \sin (\theta))^{2} \\
\left|\mathbf{k}_{\mathbf{x}}\right|^{2}=(2 \omega s(\theta) \cos (\theta))^{2} \\
\mathbf{k}_{\boldsymbol{\lambda}} \cdot \mathbf{k}_{\mathbf{x}}=0
\end{array}
$$

We can eliminate from equations 24- 25 the dependence on the depth axis and obtain an angle decomposition formulation prior to imaging. Thus, if we eliminate $k_{z}$ and $k_{\lambda_{z}}$, we obtain the expression:

$$
\begin{array}{r}
\left(k_{x}{ }^{2}+k_{y}{ }^{2}\right)(2 \omega s(\theta) \sin \theta)^{2}+\left(k_{\lambda_{x}}{ }^{2}+k_{\lambda_{y}}{ }^{2}\right)(2 \omega s(\theta) \cos \theta)^{2}= \\
\left(k_{x} k_{\lambda_{y}}-k_{y} k_{\lambda_{x}}\right)^{2}+(2 \omega s(\theta) \sin \theta)^{2}(2 \omega s(\theta) \cos \theta)^{2} .
\end{array}
$$

The quadratic equation 27 can be used to map data from space-lag gathers $\left(k_{\lambda_{x}}, k_{\lambda_{y}}\right)$ into angle coordinates $\theta$, prior to imaging. For 2D data, equation 27 takes the simpler form

$$
\begin{array}{r}
k_{x}{ }^{2}(2 \omega s(\theta) \sin \theta)^{2}+k_{\lambda_{x}}{ }^{2}(2 \omega s(\theta) \cos \theta)^{2}= \\
(2 \omega s(\theta) \sin \theta)^{2}(2 \omega s(\theta) \cos \theta)^{2}
\end{array}
$$

which can be solved for an explicit mapping of $k_{\lambda_{x}}$ to $\theta$.

Note that the angle decomposition formula 28 reduces to a form simpler than that shown by Alkhalifah and Fomel (2009) for VTI media. This angle decomposition is particularly useful in imaging via downward continuation, as discussed next.

\section{DOWNWARD CONTINUATION}

The angle decomposition discussed in the preceding section allows us to produce angle gathers after downward continuation in DTI media. Wavefield reconstruction for multioffset migration based on the one-way wave-equation under the survey-sinking framework (Claerbout, 1985) is implemented by recursive phase-shift of prestack wavefields

$$
u_{z+\Delta z}(\mathbf{m}, \mathbf{h})=e^{-i k_{z} \Delta z} u_{z}(\mathbf{m}, \mathbf{h})
$$

followed by extraction of the image at time $t=0$. Here, $\mathbf{m}$ and $\mathbf{h}$ represent the midpoint and half-offset coordinates, which are equivalent with the space and space-lag variables discussed earlier, but restricted to the horizontal plane. In equation $29, u_{z}(\mathbf{m}, \mathbf{h})$ represents the acoustic wavefield for a given frequency $\omega$ at all midpoint positions $\mathbf{m}$ and half-offsets $\mathbf{h}$ at depth $z$, and $u_{z+\Delta z}(\mathbf{m}, \mathbf{h})$ represents the same wavefield extrapolated to depth $z+\Delta z$. The phase shift operation uses the depth wavenumber $k_{z}$ which is defined in 2D by the DSR equation 4 as follows:

$$
k_{z}=\sqrt{\omega^{2} s^{2}(\theta)-\left(k_{m}-k_{h}\right)^{2}}+\sqrt{\omega^{2} s^{2}(\theta)-\left(k_{m}+k_{h}\right)^{2}},
$$


where $k_{h}$ is equivalent to $k_{\lambda_{x}}$.

Figure 4 shows $k_{z}$ as a function of the midpoint wavenumber and the reflection angle for a DTI model characterized by $\eta=0.3$ (left). As expected, the range of angles reduces with increasing dip angle (or $k_{m}$ ). The phase shift per depth is maximum for horizontal reflector $\left(k_{m}=0\right)$ and zero offset (equivalent with $\theta=0$ ). The right plot in Figure 4 shows the difference between the $k_{z}$ for this DTI model and that for an isotropic model with velocity equal to $v=1.8 \mathrm{~km} / \mathrm{s}$. As expected, for zero reflection angle, the DTI phase shift is given by the isotropic operator as we discussed earlier. For the non-zero-offset case, the difference increases with the reflection angle.

To use $k_{z}$ in this form we need to evaluate the reflection angle, $\theta$, in the downward continuation process as the angle gather defines the phase angle needed for equation 30 . Equation 28 provides a one-to-one relation between angle gathers and the offset wavenumber. However, to insure an explicit evaluation we formulate the problem as a mapping process to find the wavefield for a given offset wavenumber $k_{h}$ that corresponds to a particular reflection angle. As a result, we can devise an algorithm for downward continuation for a wavefield with sources and receivers at depth $z$ as follows:

- For a given reflection angle, use equation 28 to find the corresponding $k_{h}\left(=k_{\lambda_{x}}\right)$.

- Using $k_{h}(\theta)$, map $u\left(k_{m}, k_{h}, \omega, z\right)$ to $u\left(k_{m}, \theta, \omega, z\right)$ (the angle decomposition).

- Apply the imaging condition by summing over frequencies $\omega$ to obtain imaged angle gathers.

- Apply phase shift to the wavefield $u\left(k_{m}, k_{h}, \omega, z\right)$ to obtain $u\left(k_{m}, k_{h}, \omega, z+\Delta z\right)$ by equation 29 using the depth wavenumber given by equation 30 .

- Repeat the steps for depth $z=z+\Delta z$.

The process provides imaged angle gathers in DTI media. This approach also allows us to better treat illumination as we downward continue while keeping the sampling in reflection angle uniform.

\section{DOMAIN OF APPLICABILITY}

The reflector dip TI tilt constraint introduced here for imaging simplification purposes is not applicable everywhere. In fact, setting such a constraint inherently suggests smooth interfaces as it is impossible to impose such a constraint on a diffractor. The smooth interface is also required by the plane wave assumption used in the angle gather development. Thus, we are suggesting DTI as a model development tool in which this suggested assumption is typically used in areas like the Gulf of Mexico. Thus, the DTI model must be extracted from reflections that adhere to this constraint, which do not include salt flanks. This is convenient in building the model around the salt and even subsalt. While the top-of-salt reflections do not adhere to this constraint, the bottom reflections do as isotropy is a special 

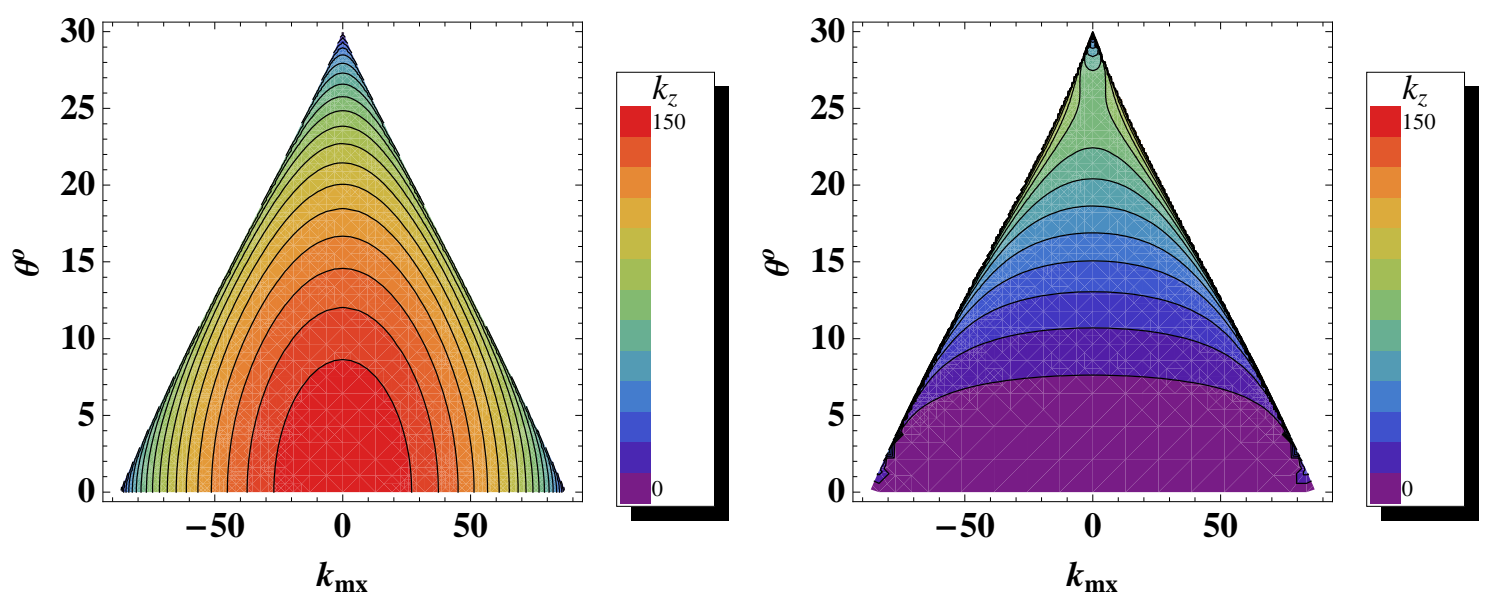

Figure 4: A plot of the vertical wavenumber, $k_{z}$, as a function of midpoint wavenumber and angle gather for a dip-constrained transversely isotropic (DTI) model with 2NMO velocity, $v=2.0 \mathrm{~km} / \mathrm{s}$, titled direction velocity, $v_{T}=1.8 \mathrm{~km} / \mathrm{s}$, and $\eta=0.3$ (left) and the difference in $k_{z}$ between the DTI model and an isotropic model with $v=1.8 \mathrm{~km} / \mathrm{s}$ (right). The wave numbers are given in units of $\mathrm{km}^{-1}$.

case of DTI with the anisotropy parameters $\eta=\delta=0$. In addition, subsalt reflections also satisfy this constraint whether such reflections are within isotropic media or an assumed DTI condition. It does not matter that the rays may have traveled through media that is VTI or general TTI, what matters is the behavior at the reflection point for applications like DTI imaging or angle gather analysis.

\section{CONCLUSIONS}

Constraining the tilt of a transversely isotropic medium normal to the reflector dip (DTI) allows for explicit formulations of plane waves around the scattering point. These formulations form the basis for angle decomposition or the moveout analysis in the extended image condition domain. As a result, DTI is a convenient model for anisotropy parameter estimation in media in which such models are applicable. This model also allows us to use the general TTI assumption in a simplified form that better fits the information embedded in the recorded data. 


\section{ACKNOWLEDGMENTS}

We are grateful to KAUST and to the sponsors of the Center for Wave Phenomena at Colorado School of Mines for their support. We thank the associate editor and the reviewers for their critical review of the letter.

\section{REFERENCES}

Alkhalifah, T., 1997, Seismic data processing in vertically inhomogeneous TI media: Geophysics, 62, 662-675.

,- 1998 , Acoustic approximations for processing in transversely isotropic media: Geophysics, 63, 623-631.

Alkhalifah, T., and J. Bednar, 2000, Building a 3-D anisotropic model: Its implications to traveltime calculation and velocity analysis: 70th Ann. Internat. Mtg, Soc. of Expl. Geophys., 965-968.

Alkhalifah, T., and S. Fomel, 2009, Angle gathers in wave-equation imaging for vti media: SEG Technical Program Expanded Abstracts, 28, 2899-2903.

Alkhalifah, T., and K. Larner, 1994, Migration error in transversely isotropic media: Geophysics, 59, 1405-1418.

Alkhalifah, T., and I. Tsvankin, 1995, Velocity analysis for transversely isotropic media: Geophysics, 60, 1550-1566.

Audebert, F. S., A. Pettenati, and V. Dirks, 2006, TTI anisotropic depth migration - which tilt estimate should we use?: EAGE, Expanded Abstracts, P185.

Ball, G., 1995, Estimation of anisotropy and anisotropic 3-D prestack depth migration, offshore Zaire: Geophysics, 60, 1495-1513.

Berkhout, A. J., 1982, Imaging of acoustic energy by wave field extrapolation: Elsevier.

Claerbout, J. F., 1985, Imaging the Earth's interior: Blackwell Scientific Publications.

- , 1995, Basic Earth Imaging: Stanford Exploration Project.

Clayton, R. W., and R. H. Stolt, 1981, A Born-WKBJ inversion method for acoustic reflection data: Geophysics, 46, 1559-1567.

Goldin, S. V., 1986, Seismic Traveltime Inversion: Soc. of Expl. Geophys.

Huang, T., S. Xu, J. Wang, G. Ionescu, and M. Richardson, 2008, The benefit of tti tomography for dual azimuth data in gulf of mexico: SEG, Expanded Abstracts, 27, 222-226.

Isaac, J. H., and D. C. Lawton, 2004, A practical method for estimating effective parameters of anisotropy from reflection seismic data: Geophysics, 69, 681-689.

Martinez, R. D., and S. Lee, 2002, A strategy for anisotropic P-wave prestack imaging: SEG, Expanded Abstracts, 21, 149-152.

Ohlsen, F., and C. MacBeth, 1999, Elliptical anisotropy: Regression or advance?: SEG, Expanded Abstracts, 18, 1600-1603.

Rickett, J., and P. Sava, 2002, Offset and angle-domain common image-point gathers for shot-profile migration: Geophysics, 67, 883-889.

Sava, P., and S. Fomel, 2005, Coordinate-independent angle-gathers for wave equation migration: 75th Annual International Meeting, SEG, Expanded Abstracts, 2052-2055. 
_, 2006 , Time-shift imaging condition in seismic migration: Geophysics, 71, S209_ S217.

Stolt, R. H., and A. B. Weglein, 1985, Migration and inversion of seismic data: Geophysics, 50, 2458-2472.

Weglein, A. B., and R. H. Stolt, 1999, Migration-inversion revisited (1999): The Leading Edge, 18, 950-952.

Yang, T., and P. Sava, 2009, Moveout analysis of wave-equation extended images: Geophysics, submitted for publication.

Zhou, H., D. Pham, S. Gray, and B. Wang, 2004, Tomographic velocity analysis in strongly anisotropic tti media: SEG, Expanded Abstracts, 23, 2347-2350. 